\title{
Dissecting the genomic complexity underlying medulloblastoma
}

\author{
A list of authors and their affiliations appears at the end of the paper
}

Medulloblastoma is an aggressively growing tumour, arising in the cerebellum or medulla/brain stem. It is the most common malignant brain tumour in children, and shows tremendous biological and clinical heterogeneity ${ }^{1}$. Despite recent treatment advances, approximately $40 \%$ of children experience tumour recurrence, and $30 \%$ will die from their disease. Those who survive often have a significantly reduced quality of life. Four tumour subgroups with distinct clinical, biological and genetic profiles are currently identified ${ }^{2,3}$. WNT tumours, showing activated wingless pathway signalling, carry a favourable prognosis under current treatment regimens ${ }^{4}$. SHH tumours show hedgehog pathway activation, and have an intermediate prognosis ${ }^{2}$. Group 3 and 4 tumours are molecularly less well characterized, and also present the greatest clinical challenges ${ }^{2,3,5}$. The full repertoire of genetic events driving this distinction, however, remains unclear. Here we describe an integrative deep-sequencing analysis of 125 tumour-normal pairs, conducted as part of the International Cancer Genome Consortium (ICGC) PedBrain Tumor Project. Tetraploidy was identified as a frequent early event in Group 3 and 4 tumours, and a positive correlation between patient age and mutation rate was observed. Several recurrent mutations were identified, both in known medulloblastoma-related genes (CTNNB1, PTCH1, MLL2, SMARCA4) and in genes not previously linked to this tumour $(D D X 3 X, C T D N E P 1, K D M 6 A, T B R 1)$, often in subgroup-specific patterns. RNA sequencing confirmed these alterations, and revealed the expression of what are, to our knowledge, the first medulloblastoma fusion genes identified. Chromatin modifiers were frequently altered across all subgroups. These findings enhance our understanding of the genomic complexity and heterogeneity underlying medulloblastoma, and provide several potential targets for new therapeutics, especially for Group 3 and 4 patients.

As a first phase of the International Cancer Genome Consortium (ICGC) PedBrain Tumor Project (http://www.pedbraintumor.org), we have collected matched tumour and germline samples from 125 medulloblastoma patients aged from 0 to 17 years (Supplementary Table 1). Whole-genome sequencing (WGS, $n=39$ ) and whole-exome sequencing (WES, $n=21$ ) were applied to a 'discovery' set, with a custom-capture approach used to sequence 2,734 genes in an additional 'replication' set $(n=65)$. All tumour samples were obtained at primary diagnosis, before adjuvant therapy, and the distribution of molecular subgroups was similar across cohorts (Supplementary Fig. 1).

Investigation of genome-wide somatic mutation allele frequencies identified several cases with a clear peak at approximately $25 \%$, rather than the expected approximately 50\% allele frequency for early, heterozygous events (Fig. 1a). Analysis of coverage depth and allele frequencies in regions of copy-number change ruled out stromal contamination, but rather indicated a tetraploid baseline in the tumour genome (Fig. 1b). Predicted ploidy status was confirmed by fluorescence in situ hybridization (FISH) using multiple centromeric probes in 17 out of 18 cases analysed (Fig. 1a). The extremely low fraction of mutations at approximately $50 \%$ allele frequency indicates that genome duplication occurred very early during tumorigenesis.
Some cases probably went through even higher polyploidy states before reaching an approximately $4 \mathrm{n}$ baseline (for example ICGC_MB45, displaying 4n chromosomes with 4:0 or 3:1 allele ratios; Supplementary Fig. 2). Across the discovery set, tetraploidy was most commonly observed in Group 3 (7 out of 13,54\%) and Group 4 tumours ( 8 out of $20,40 \%$ ), followed by SHH (4 out of $14,29 \%$ ) and WNT tumours (1 out of 7, 14\%). Interestingly, the four tetraploid SHH tumours all harboured TP53 mutations and also displayed chromothripsis ${ }^{6}$. Tetraploid Group 3 and 4 tumours showed significantly more large-scale copy number alterations compared with diploid cases (median 10 changes per tumour in tetraploid versus 4 per tumour in diploid cases, $P=0.008$, two-tailed Mann-Whitney $U$-test; Supplementary Fig. 3). Thus, tetraploidy followed by genomic instability may be an early driving event in a large proportion of Group 3 and 4 medulloblastomas, which pose a significant clinical challenge due to their dismal prognosis and lack of targeted treatment options. Novel classes of drugs such as mitotic checkpoint kinase or kinesin inhibitors, which target the maintenance of tetraploidy through successive cell divisions, may therefore represent a rational therapeutic strategy in these cases $^{7,8}$. The value of tetraploidy as a prognostic marker also requires further investigation.

The average somatic mutation rate in the WGS cohort was 0.52 per megabase $(\mathrm{Mb})$, with an average of 10.3 non-synonymous coding single-nucleotide variants (SNVs) in the discovery cohort (Supplementary Table 2). This is slightly higher than previously reported for medulloblastoma ${ }^{9}$, possibly due to improved coverage and technical sensitivity, but considerably lower than in deep-sequenced adult tumours, for example $e^{10,11}$. There were significantly fewer transitions in the somatic alterations compared with germline variation $\left(P=4.6 \times 10^{-7}\right.$, Wilcoxon rank-sum test; Supplementary Fig. 4). All coding somatic SNVs identified in the combined cohort are listed in Supplementary Table 3.

We identified a positive correlation between genome-wide mutation rate and patient age, as previously reported for coding mutations ${ }^{9}$ $\left(r^{2}=0.35, P=7.8 \times 10^{-5}\right.$ Pearson's product-moment correlation; Fig. 1c). Intriguingly, this association was more pronounced in diploid tumours $\left(r^{2}=0.52, P=3 \times 10^{-5}\right)$, and virtually absent in tetraploid cases $\left(r^{2}=0.04, P=0.5\right)$ (Supplementary Fig. 5a, b). A similar trend was observed for non-synonymous mutations across the discovery cohort (Supplementary Fig. 5c). Coverage level did not correlate with mutation rate (Supplementary Fig. 5d). One explanation may be that all medulloblastomas originate during embryogenesis, with some tumours needing to accumulate more genetic 'hits' before becoming symptomatic. Alternatively, tumours arising in older patients may derive from more differentiated cells that require a greater number of alterations to undergo malignant transformation. Investigation of additional tumours from older patients may help to clarify this.

Five SHH tumours harbouring TP53 mutations, including three previously described Li-Fraumeni syndrome (LFS)-associated tumours with germline mutations ${ }^{6}$, one newly identified LFS case (ICGC_MB23), and one somatically mutated tumour (ICGC_MB34), had significantly more mutations than the remaining cases, both genome wide (mean 1.1 per $\mathrm{Mb}$ versus 0.43 per $\mathrm{Mb}, P=4.5 \times 10^{-6}$; two-tailed $t$-test) and for 

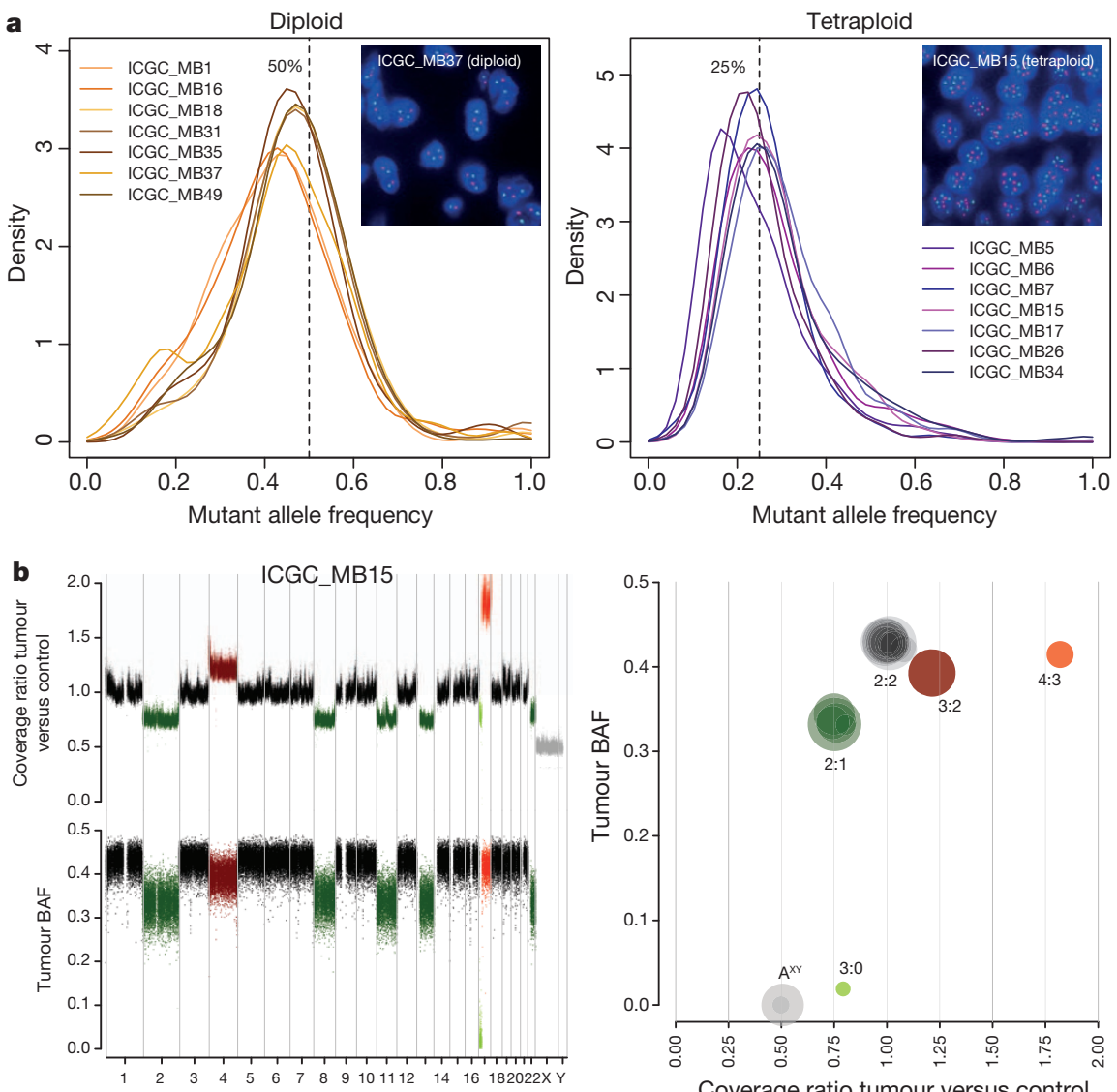

Chromosome

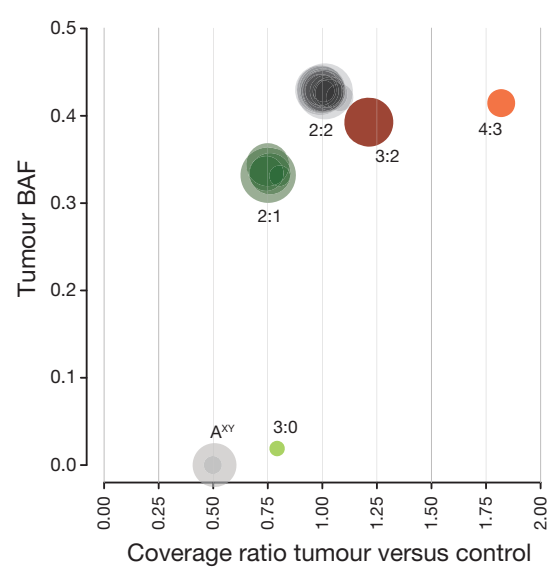

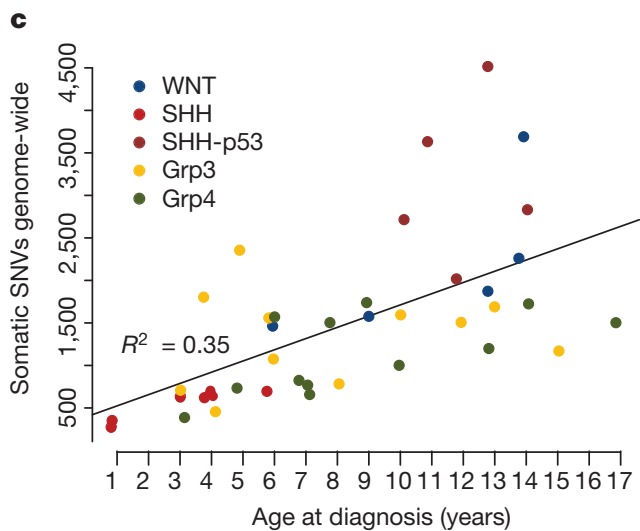

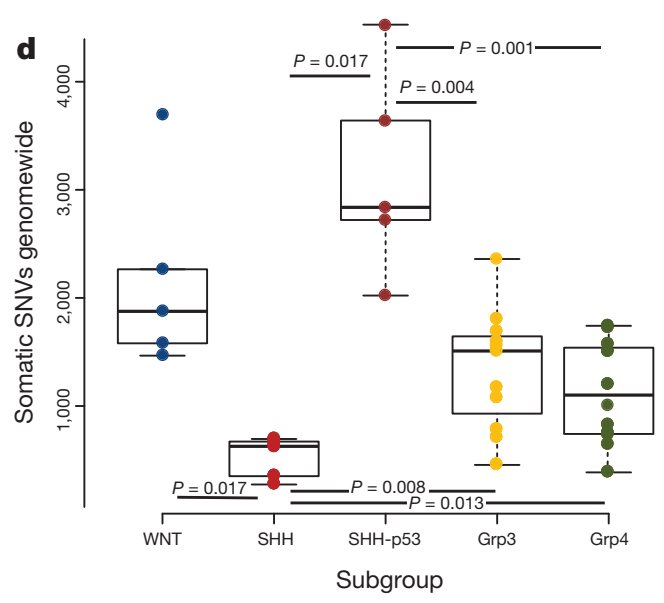

Figure $1 \mid$ Tetraploidy is a frequent early event in medulloblastoma tumorigenesis, and mutation rates vary with age and subgroup. a, Distributions of genome-wide somatic mutation allele frequencies (the proportion of sequence reads supporting a mutation) for diploid tumours (with a peak at $\sim 50 \%$ for heterozygous events, $n=7$ ) and tetraploid cases (with a peak at $\sim 25 \%, n=7$ ). Insets show centromeric FISH for chromosomes 1 (red) and 11 (green), confirming the predicted ploidy status. b. Top left, rescaled tumour:germline coverage ratio, indicating copy-number gains (red) or losses (green). Bottom left, B-allele frequency (BAF) in the tumour at SNP positions

non-synonymous changes (mean 23 versus $8.8, P=2.6 \times 10^{-6}$ ). Interestingly, the WNT subgroup, which typically shows a good prognosis and few copy-number changes, had the next highest mutation rate (Fig. 1d).

Forty-one somatic, coding, small insertions/deletions (Indels) were identified across the cohort, with an average of 0.4 coding Indels per case in the discovery set (range $0-2$; Supplementary Table 4 ). Some genes, however, were more commonly affected by Indels than SNVs. For example, frameshift Indels in PTCH1 were detected in 6 out of 125 cases, whereas only 2 SNVs were observed. Recurrent Indels were also seen in the chromatin modifiers MLL2, KDM6A (3 cases each) and $B C O R$ ( 2 cases).

In contrast to another paediatric brain tumour, glioblastoma, in which we recently identified frequently recurrent hotspot mutations ${ }^{12}$, the majority of mutated genes in this study were unique to a single case (587 out of 760 non-synonymous SNVs in the 125 cases, 77\%), demonstrating the pronounced genetic heterogeneity of medulloblastoma. Twenty-five of these singleton mutations, and $53 \mathrm{SNVs}$ in total, were at positions listed in the COSMIC database of somatic alterations in tumours (available at http://www.sanger.ac.uk/genetics/CGP/ cosmic/), suggesting a rare but important contribution of many known cancer genes in medulloblastoma (Supplementary Table 5). Only 8 genes were somatically altered in more than $3 \%$ of the whole series: CTNNB1 (15 cases, $12 \%) ; D D X 3 X$ (10 cases, $8 \%$ ); PTCH1 ( 8 cases, $6 \%$ ), SMARCA4 (6 cases, 5\%), MLL2 (6 cases, 5\%), TP53 (somatically which are heterozygous in the germ line. Right, genome alteration print (GAP) of segmented copy number and allele frequency profiles. Chromosomes with predicted 3:0/2:1/3:2 allele ratios show a BAF of approximately $0 / 0.33 / 0.4$ and coverage ratios of approximately $0.75 / 0.75 / 1.25$. Owing to random sampling, the 2:2 allele ratio is slightly below 0.5 . c, Genome-wide somatic mutation rates are positively correlated with patient age $(n=39)$. Grp, Group. d, Distribution of somatic mutation rates by tumour subgroup $(n=39)$. $P$ values are according to a Wilcoxon rank-sum test with Bonferroni correction. SHH-p53, SHHsubgroup tumours harbouring a somatic or germline TP53 mutation.

mutated in 5 cases, $4 \%$ ), KDM6A ( 5 cases, $4 \%$ ) and CTDNEP1 ( 4 cases, $3 \%$ ) (Fig. 2). These were also the only genes found to be significantly altered upon analysis of the combined cohort with MutSig, an algorithm testing whether the observed mutations in a gene are not simply a consequence of random background mutation processes. It takes into account gene length and composition, silent to non-silent mutation ratios, and other factors (see https://confluence.broadinstitute. org/display/CGATools/MutSig; Supplementary Table 6). Large-scale copy-number changes known to be associated with medulloblastoma, such as formation of an isodicentric $17 \mathrm{q}$ and losses of $10 \mathrm{q} / 9 \mathrm{q} / \mathrm{X}^{13-15}$, were more frequently recurrent than SNVs (Supplementary Fig. 6a-e).

Many alterations were enriched in specific medulloblastoma subgroups. For example, all of the WNT tumours (15 out of 15) harboured a mutation in CTNNB1, and 13 out of 15 displayed loss of one copy of chromosome 6 (or acquired uniparental disomy in one case), alterations which have previously been associated with this subgroup ${ }^{4,13,15}$. Mutations in DDX3X were also clearly enriched in WNT tumours (adjusted $P=7.06 \times 10^{-6}$, two-tailed Fisher's exact test with a Bonferroni correction), and these mutations were clustered within the helicase domain (Supplementary Fig. 7a). Three were localized at the RNA-binding surface of the protein and three were predicted to disrupt the closed (RNA-binding) conformation (Supplementary Fig. 7b). The remainder were predicted to disrupt indirectly either the positive charge on the RNA-binding surface $(n=2)$ or the folding of the closed form $(n=2)$. No truncating mutations were found, 

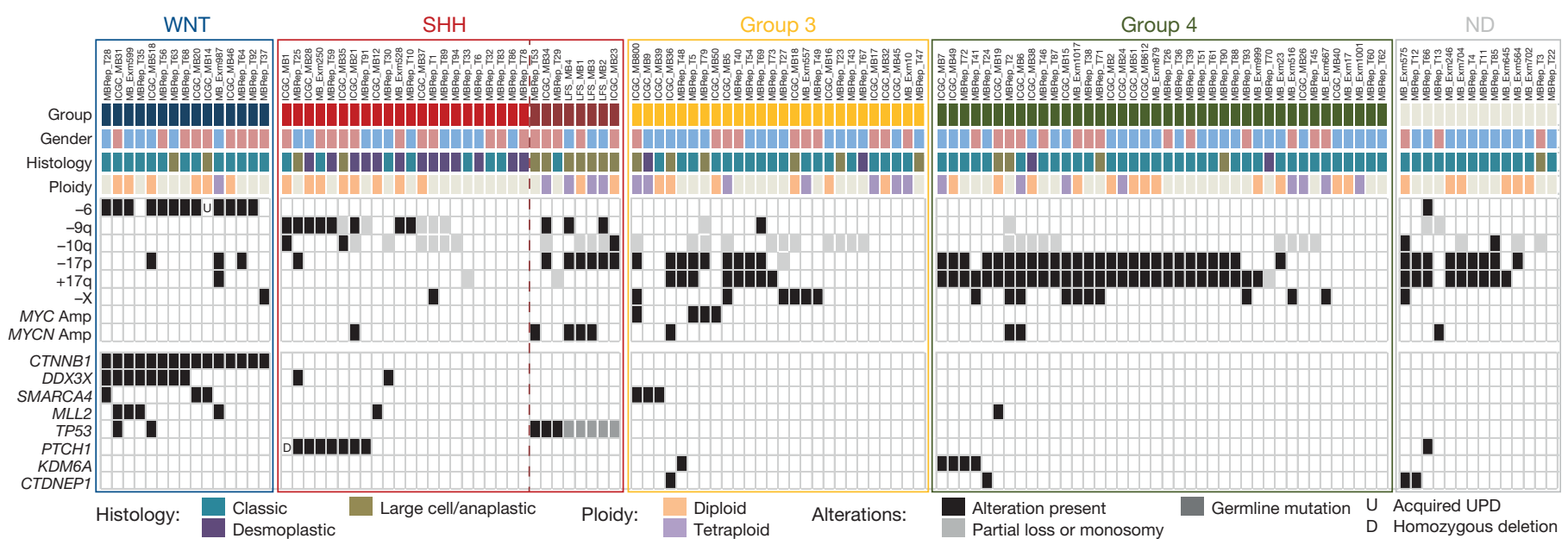

Figure $2 \mid$ Subgroup specificity of common genetic alterations. Summary of clinical data and recurrent alterations in the combined cohort $(n=125)$. Genes which were found to be significantly mutated by MutSig analysis were included.

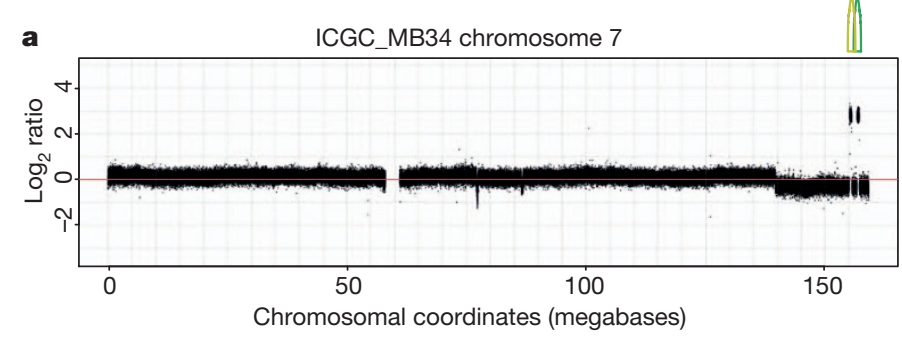

b

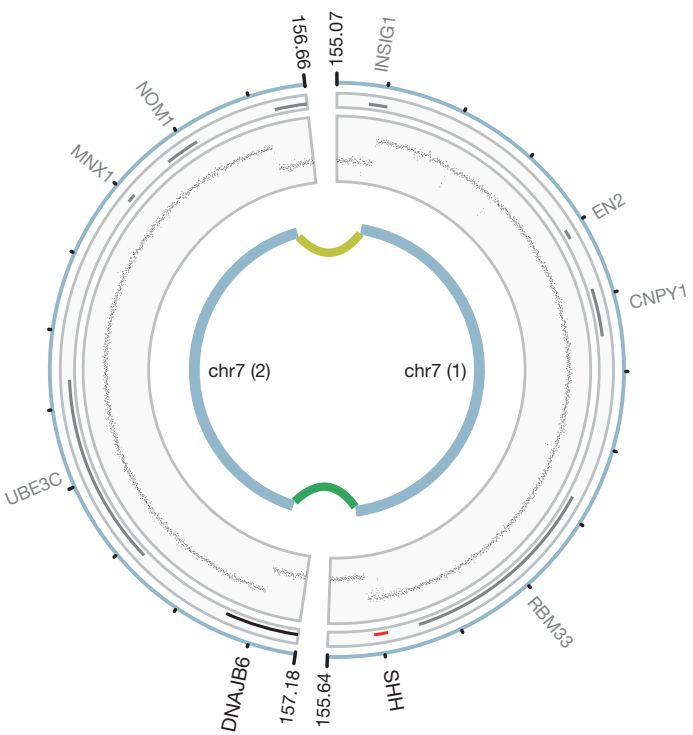

c

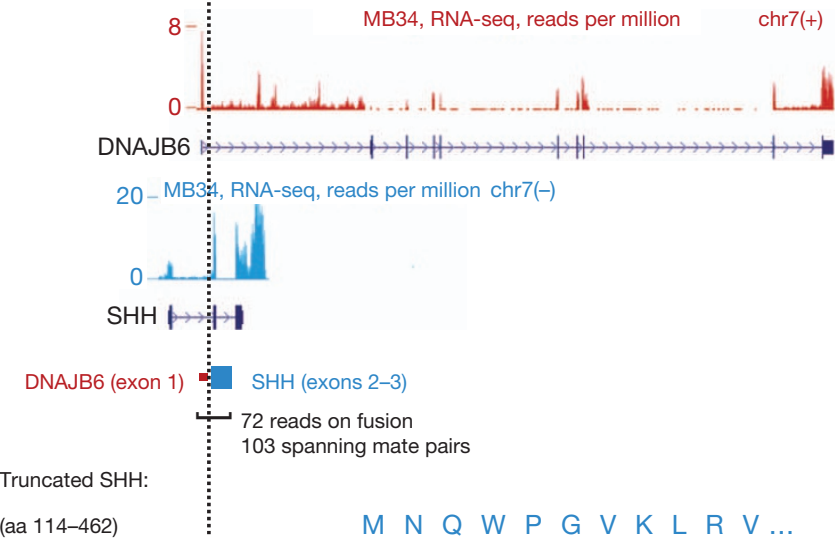

UPD, uniparental disomy; ND, no material available for conclusive molecular subgroup assignment.

indicating an alteration rather than simply a loss of function. DDX3X has recently been proposed to have an oncogenic role ${ }^{10,11}$, although its exact function in tumorigenesis remains to be determined.

As anticipated from previous studies ${ }^{13,16}$, SHH tumours frequently showed loss of the whole of chromosome arm $9 \mathrm{q}$, as well as alterations in key hedgehog-pathway signalling molecules (for example, $\mathrm{PTCH}$, altered in 8 cases; $M Y C N$, amplified in 5 cases; and SMO, mutated in ICGC_MB12).

The most frequently mutated gene in Group 3 tumours was SMARCA4 ( 3 out of 26 cases). As with DDX3X, these mutations were clustered in the helicase domain (Supplementary Fig. 7a). As noted above, tetraploidy was also a common event in this subgroup and in Group 4 tumours. Recurrent truncating mutations in KDM6A (on chromosome $\mathrm{X}$, which frequently shows copy-number loss in female Group 3 and 4 medulloblastoma patients; also known as UTX), encoding a histone 3 lysine 27 (H3K27) demethylase, were also seen in Group 4 (4 out of $40,10 \%$ ), indicating a tumour-suppressive role in this subgroup, as previously described for other cancers ${ }^{17}$. CTDNEP1 (a homologue of the Xenopus gene dullard), was also affected by truncating alterations in four tumours. In three of these cases, the mutation was accompanied by loss of the wild-type allele through isodicentric $17 \mathrm{q}$ formation. This gene, encoding a nuclear envelope phosphatase, was shown in Xenopus to have roles in BMP signalling and neural development ${ }^{18}$. In mammalian cells it is involved in the lipin activation pathway, regulating nuclear membrane biogenesis and production of diacylglycerol ${ }^{19,20}$. Given the high frequency of isodicentric $17 \mathrm{q}$ in medulloblastoma, genetic targets on this chromosome have long been sought after. CTDNEP1 may be a good candidate for one of the medulloblastoma tumour suppressors on $17 \mathrm{p}$.

Aside from these subgroup-enriched events, a commonly recurring theme across all medulloblastomas is alterations in genes involved in chromatin modification. Some point mutations and DNA copy number alterations in this pathway have previously been implicated in medulloblastoma ${ }^{9,21}$. Overall, 45 out of 125 cases (36\%) harboured a mutation in a gene categorized under the Gene Ontology term 'Chromatin Modification' (GO:0015168, Supplementary Fig. 6f, g).

We recently described an enrichment of catastrophic DNA rearrangements ('chromothripsis') in TP53-mutated SHH medulloblasto$\mathrm{mas}^{6}$. Three new TP53-mutant SHH tumours were identified in this

Figure 3 Identification of novel fusion genes in medulloblastoma. a, Readdepth plot with $\log _{2}$ tumour:germline coverage ratio showing alterations on chromosome 7 in ICGC_MB34. Lines indicate connected segments. b, Schematic of the rearrangement. c, Details of the $S H H$ fusion gene structure and support for its expression, derived from RNA sequencing data. aa, amino acids. 
study: ICGC_MB23 (germline mutation), MBRep_T29 and MBRep_T53 (somatic mutations). Two of these, ICGC_MB23 and MBRep_T53, showed complex genomic rearrangements indicative of the chromothripsis model (Supplementary Fig. 8) (22. $^{22}$

Deep sequencing also allowed fine mapping of two amplicons on chromosome 7 in ICGC_MB34 (a SHH tumour with a somatic TP53 mutation, relating to MB2034 in ref. 6). One amplicon included the entire $S H H$ gene, whereas the second disrupted DNAJB6, such that its first exon was juxtaposed to $S H H$ (Fig. 3a, b). RNA sequencing further revealed a novel fusion transcript, not expected from the DNA data, containing the first exon of DNAJB6 and exons 2 and 3 of SHH. The first exon of $\mathrm{SH} H$ was skipped, resulting in a predicted amino-terminally truncated $\mathrm{SHH}$ protein (Fig. 3c). Expression of SHH was extremely high in this case, although virtually absent in 301 other medulloblastomas (Supplementary Fig. 9a). Predicted DNA and RNA junctions were validated by PCR (Supplementary Fig. 9b).

Several additional in-frame gene fusions were identified by large insert mate-pair sequencing, which gives better resolution for structural variant detection. ICGC_MB18, for example, carried an intrachromosomal translocation resulting in a fusion between LCLAT1 and $E R B B 4$, the latter of which has previously been associated with medulloblastoma oncogenesis ${ }^{23}$ (Supplementary Fig. 9c-f). In ICGC_MB6, a complex rearrangement of fragments from chromosomes 1 and 17 produced a fusion between MLLT6 and MRPL45, a mitochondrial ribosomal protein, resulting in strong overexpression of the latter (Supplementary Fig. 10a-c). These findings indicate that gene fusions involving well-established medulloblastoma oncogenes may have a more important role in medulloblastoma than previously recognized, and warrant further investigation.

High-coverage, strand-specific RNA sequencing of 28 cases allowed us to determine the proportion of DNA SNVs that were observable in the transcriptome (Supplementary Tables 3 and 4). Overall, 129 out of 268 (48\%) non-synonymous mutations in the DNA were also detectable at the RNA level. A further 38\% (101 out of 268) resided in genes expressed at extremely low abundance (reads per kilobase of exon model per million mapped reads $(\mathrm{RPKM})<1$ ). Thus, the fraction of expressed mutations is even smaller than the already low number of DNA alterations, supporting the hypothesis that very few driving hits are needed to generate this paediatric tumour. It may also be the case that some mutations required for tumour initiation are not essential for later tumour cell maintenance.

RNA sequencing further revealed monoallelic expression of a heterozygous mutation in TBR1, producing a p.G275C change, which was also seen in a previous study ${ }^{9}$ (Supplementary Fig. 11a). TBR1 encodes a T-box transcription factor involved in brain development ${ }^{24}$. This gene, and a second family member, EOMES (or TBR2), clearly showed subgroup-specific differential expression (Fig. 4a). Sequencing of TBR 1 exon 2 in a further 85 medulloblastomas revealed one additional case with an identical mutation. All three mutated tumours were in Group 4. Gene expression was also strongly correlated with DNA methylation for both TBR1 and EOMES (Fig. 4b, c and Supplementary Fig. 11b, c), and expression of TBR1 and EOMES is inversely correlated a
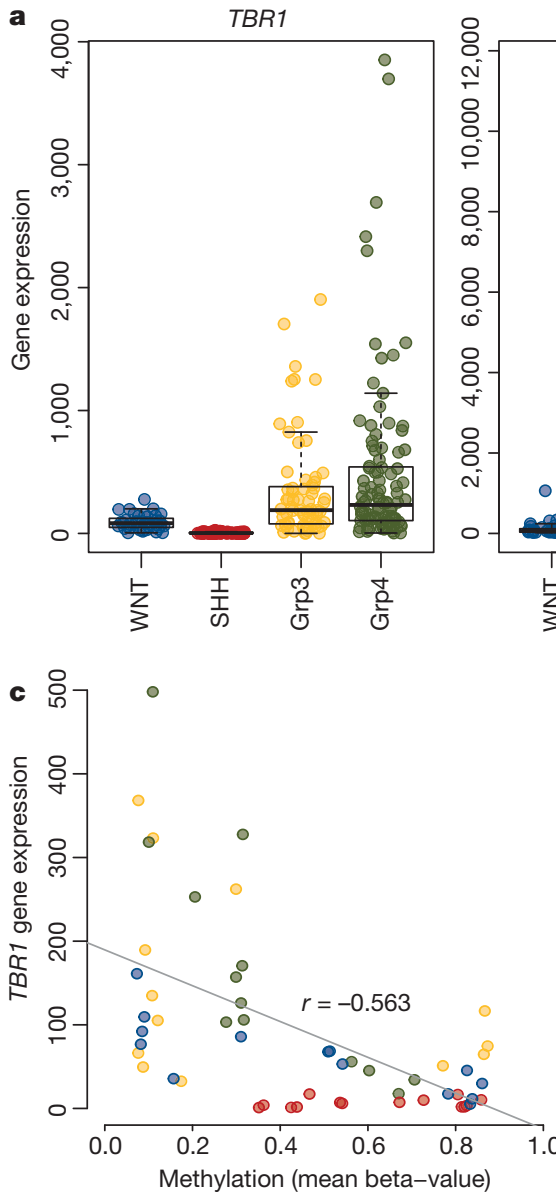

Figure 4 Integration of mutation, expression and methylation data shows differential regulation of $T B R 1$ and $E O M E S$ in medulloblastoma. a, Microarray data showing clear differences in TBR1 and EOMES expression between medulloblastoma subgroups $(n=301)$. $\mathbf{b}$, DNA methylation of TBR1 $(n=54)$, ranging from low (blue) to high (red). Horizontal red bar indicates the region used for correlation analysis in c. c, Expression of TBR1 is tightly

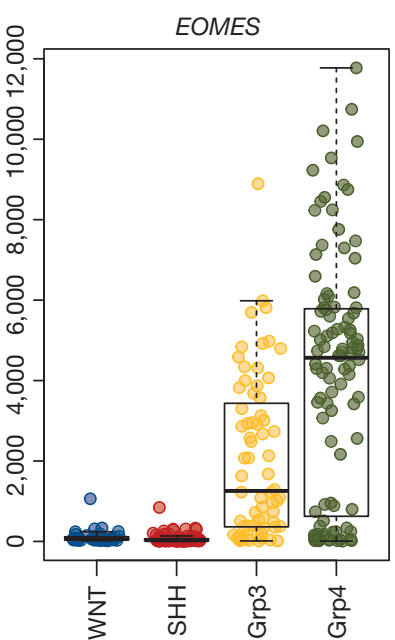

b Chromosome 2

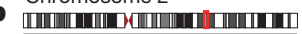
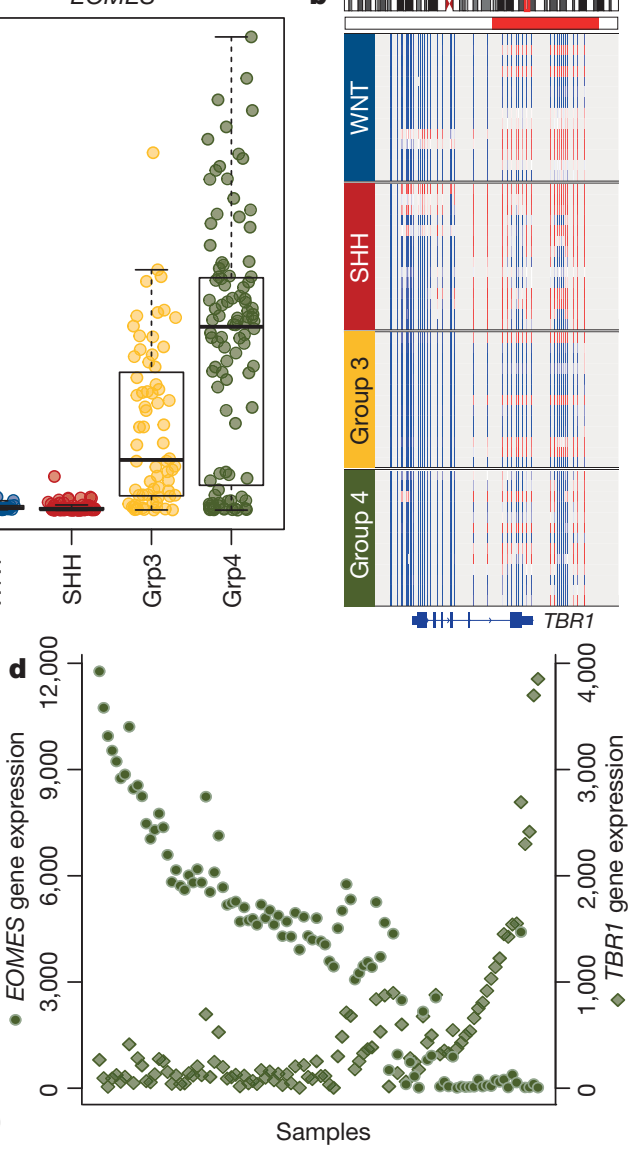

correlated with gene methylation ( $n=54$; Pearson's correlation values, $r$ ). SHH tumours show high methylation and virtually no expression, whereas WNT, Group 3 and Group 4 tumours display a more varied pattern. d, Expression levels of TBR1 (diamonds) and EOMES (circles) are inversely related in Group 4 tumours $(n=104)$ 
in Group 4 tumours (Fig. 4d), giving subsets that are either TBR1methylated and EOMES ${ }^{\text {hi }}$ or EOMES-methylated and TBR1 ${ }^{\text {hi }}$ (Supplementary Fig. 11d, e). These two genes are markers for different stages of neuronal lineage commitment, suggesting possible differences in cell-of-origin or differentiation within Group 4 subpopulations ${ }^{25}$.

This large, integrative genomics study has provided a detailed insight into new mechanisms contributing to medulloblastoma tumorigenesis and disclose novel targets for therapeutic approaches, especially for Group 3 and 4 patients. The molecular subgroup-related enrichment of many alterations highlights the importance of considering this distinguishing factor in research, trial design and clinical practice.

\section{METHODS SUMMARY}

All patient material was collected after receiving informed consent according to ICGC guidelines and as approved by the institutional review board of contributing centres. Tumour subgrouping was based on gene expression profiling or immunohistochemical analysis as described in ref. 5.

Next generation sequencing was performed using Illumina technologies. Mean DNA sequence coverage was 35 -fold for whole-genome cases (range 26-56 $\times$ ) whereas mean on-target coverage in the whole-exome and replication cohorts was 68 -fold ( $74 \%$ of targets above $20 \times$ for whole exome, $66 \%$ for the replication cohort). Exome capture was carried out with Agilent SureSelect (Human All Exon $50 \mathrm{Mb}$ and XT Custom Library) in-solution reagents. Sequence data were aligned to the hg19 human reference genome assembly; duplicate and nonuniquely mapping reads were excluded. Tumour ploidy was predicted from sequencing data by a novel approach integrating copy number aberrations with allele frequencies. A subset of sequence variants were validated using PCR and Sanger sequencing. Verification rates were 95\% (128 out of 135) for SNVs and $100 \%$ (14 out of 14) for Indels (Supplementary Tables 3 and 4). A complete description of the materials and methods is provided in the Supplementary Information.

\section{Received 3 February; accepted 6 June 2012.}

\section{Published online 25 July 2012.}

1. Louis, D. N. et al. The 2007 WHO classification of tumours of the central nervous system. Acta Neuropathol. 114, 97-109 (2007)

2. Kool, M. et al. Molecular subgroups of medulloblastoma: an international metaanalysis of transcriptome, genetic aberrations, and clinical data of WNT, SHH, Group 3, and Group 4 medulloblastomas. Acta Neuropathol. 123, 473-484 (2012).

3. Taylor, M. D. et al. Molecular subgroups of medulloblastoma: the current consensus. Acta Neuropathol. 123, 465-472 (2012).

4. Clifford, S. C. et al. Wnt/Wingless pathway activation and chromosome 6 loss characterise a distinct molecular sub-group of medulloblastomas associated with a favourable prognosis. Cell Cycle 5, 2666-2670 (2006).

5. Northcott, P. A. et al. Medulloblastoma comprises four distinct molecular variants. J. Clin. Oncol. 29, 1408-1414 (2011).

6. Rausch, T. et al. Genome sequencing of pediatric medulloblastoma links catastrophic DNA rearrangements with TP53 mutations. Cell 148, 59-71 (2012).

7. Rello-Varona, S. et al. Preferential killing of tetraploid tumor cells by targeting the mitotic kinesin Eg5. Cell Cycle 8, 1030-1035 (2009)

8. Vitale, l. et al. Inhibition of Chk1 kills tetraploid tumor cells through a p53dependent pathway. PLOS ONE 2, e1337 (2007)

9. Parsons, D. W. et al. The genetic landscape of the childhood cancer medulloblastoma. Science 331, 435-439 (2011)

10. Stransky, N. et al. The mutational landscape of head and neck squamous cell carcinoma. Science 333, 1157-1160 (2011)

11. Wang, L. et al. SF3B1 and other novel cancer genes in chronic lymphocytic leukemia. N. Engl. J. Med. 365, 2497-2506 (2011)

12. Schwartzentruber, J. et al. Driver mutations in histone $\mathrm{H} 3.3$ and chromatin remodelling genes in paediatric glioblastoma. Nature 482, 226-231 (2012).

13. Kool, M. et al. Integrated genomics identifies five medulloblastoma subtypes with distinct genetic profiles, pathway signatures and clinicopathological features. PLOS ONE 3, e3088 (2008).

14. Pfister, S. et al. Outcome prediction in pediatric medulloblastoma based on DNA copy-number aberrations of chromosomes $6 q$ and $17 q$ and the MYC and MYCN loci. J. Clin. Oncol. 27, 1627-1636 (2009).

15. Thompson, M. C. et al. Genomics identifies medulloblastoma subgroups that are enriched for specific genetic alterations. J. Clin. Oncol. 24, 1924-1931 (2006).

16. Pietsch, T. et al. Medulloblastomas of the desmoplastic variant carry mutations of the human homologue of Drosophila patched. Cancer Res. 57, 2085-2088 (1997)

17. van Haaften, G. et al. Somatic mutations of the histone H3K27 demethylase gene UTX in human cancer. Nature Genet. 41, 521-523 (2009).

18. Satow, R., Kurisaki, A., Chan, T. C., Hamazaki, T. S. \& Asashima, M. Dullard promotes degradation and dephosphorylation of BMP receptors and is required for neural induction. Dev. Cell 11, 763-774 (2006).
19. Han, S. et al. Nuclear envelope phosphatase 1-regulatory subunit 1 (formerly TMEM188) is the metazoan Spo7p ortholog and functions in the lipin activation pathway. J. Biol. Chem. 287, 3123-3137 (2012)

20. Kim, Y. et al. A conserved phosphatase cascade that regulates nuclear membrane biogenesis. Proc. Natl Acad. Sci. USA 104, 6596-6601 (2007).

21. Northcott, P. A. etal. Multiple recurrent genetic events converge on control of histone lysine methylation in medulloblastoma. Nature Genet. 41, 465-472 (2009).

22. Stephens, P. J. et al. Massive genomic rearrangement acquired in a single catastrophic event during cancer development. Cell 144, 27-40 (2011).

23. Gilbertson, R. J., Perry, R. H., Kelly, P. J., Pearson, A. D. J. \& Lunec, J. Prognostic significance of HER2 and HER4 coexpression in childhood medulloblastoma. Cancer Res. 57, 3272-3280 (1997)

24. Hevner, R. F. et al. Tbr1 regulates differentiation of the preplate and layer 6. Neuron 29, 353-366 (2001).

25. Englund, C. et al. Pax6, Tbr2, and Tbr1 are expressed sequentially by radial glia, intermediate progenitor cells, and postmitotic neurons in developing neocortex. J. Neurosci. 25, 247-251 (2005).

Supplementary Information is linked to the online version of the paper at www.nature.com/nature.

Acknowledgements We thank GATC Biotech AG for sequencing services. For technica support and expertise we thank: B. Haase, D. Pavlinic, B. Baying, M. Wahlers, R. Lück, I. Kutschera, K. Schlangen, M. Metsger, K. Schulz, A. Nürnberger, A. Kovacsovics, M. Linser, J. C. Lindsey, S. Bailey, D. M. Pearson, the EMBL Genomics Core Facility, the EMBL High-performance Computing Core Facility and the DKFZ Genomics and Proteomics Core Facility. This work was principally supported by the PedBrain Tumor Project contributing to the International Cancer Genome Consortium (ICGC PedBrain Tumor Project, http://www.pedbraintumor.org/), funded by German Cancer Aid (109252) and the German Federal Ministry of Education and Research (BMBF, NGFN ${ }^{\text {plus }} \# 01$ GS0883). Additional support came from the German Cancer Research Center-Heidelberg Center for Personalized Oncology (DKFZ-HIPO), the Max Planck Society, the Pediatric Brain Tumor Foundation, the Italian Neuroblastoma Foundation and the Samantha Dickson Brain Tumour Trust. This study included samples provided by the UK Children's Cancer and Leukaemia Group (CCLG) as part of CCLG-approved biological study BS-2007-04.

Author Contributions D.T.W.J., M.Su., A.M.S., H.-J.W., S.B., S.P., H.C., E.P., L.S., A.W., S.H., T.T., B.R., C.C.B., M.Sch., C.v.K., V.B., R.V., S.Wo., S.Wi. and J.F. performed and/or coordinated experimental work. N. Jäger, D.T.W.J., M.K., T.Z., B.H., M.Su, T.J.P., V.Ho., T.R., H.-J.W., J.W., M.A., V.Am, M.Z., Q.W., B.L., V.Ast, C.L., J.E., R.K., P.v.S., J.K., D.Sh., M.J.B., R.B.R. and P.A.N. performed data analysis. Y.-J.C., M.Ry., M.Re., S.C., G.P.T., U.S., V.Ha., N.G.,Y.-J.K., C.M., W.R., A.U., C.H.-M., T.M., A.E.K., A.v.D.,O.W., E.M., J.R., M.E., M.U.S., M.C.F M.H., N. Jabado, S.R., A.O.v.B., D.W., S.C.C., M.G.M., V.P.C., W.S., G.R., M.D.T. and A.K. collected data and provided patient materials. D.T.W.J., N. Jäger, D.St., M.K., V.Ho., H.W., R.E., S.M.P. and P.L. prepared the initial manuscript and figures. U.D.W., H.L., B.B., G.R., M.M., S.L.P., M.-L.Y., J.O.K., R.E., A.K., S.M.P. and P.L. provided project leadership. All authors contributed to the final manuscript.

Author Information Short-read sequencing data have been deposited at the European Genome-phenome Archive (EGA, http://www.ebi.ac.uk/ega/) hosted by the EBI, under accession number EGAS00001000215. Reprints and permissions information is available at www.nature.com/reprints. This paper is distributed under the terms of the Creative Commons Attribution-Non-Commercial-Share Alike licence, and is freely available to all readers at www.nature.com/nature. The authors declare no competing financial interests. Readers are welcome to comment on the online version of this article at www.nature.com/nature. Correspondence and requests for materials should be addressed to R.E. (r.eils@dkfz-heidelberg.de), S.M.P (s.pfister@dkfz-heidelberg.de) or P.L. (m.macleod@dkfz-heidelberg.de).

David T. W. Jones ${ }^{1 *}$, Natalie Jäger ${ }^{2 *}$, Marcel Kool ${ }^{1}$, Thomas Zichner $^{3}$, Barbara Hutter $^{2}$, Marc Sultan ${ }^{4}$, Yoon-Jae Cho ${ }^{5}$, Trevor J. Pugh ${ }^{6}$, Volker Hovestadt ${ }^{7}$, Adrian M. Stütz ${ }^{3}$, Tobias Rausch ${ }^{3}$, Hans-Jörg Warnatz ${ }^{4}$, Marina Ryzhova ${ }^{8}$, Sebastian Bender ${ }^{1}$, Dominik Sturm $^{1}$, Sabrina Pleier ${ }^{1}$, Huriye Cin $^{1}{ }^{1}$, Elke Pfaff ${ }^{1}$, Laura Sieber ${ }^{1}$, Andrea Wittmann ${ }^{1}$, Marc Remke ${ }^{1}$, Hendrik Witt ${ }^{1,9}$, Sonja Hutter ${ }^{1}$, Theophilos Tzaridis ${ }^{1}$, Joachim Weischenfeldt ${ }^{3}$, Benjamin Raeder ${ }^{3}$, Meryem Avci ${ }^{4}$, Vyacheslav Amstislavskiy ${ }^{4}$, Marc Zapatka ${ }^{7}$, Ursula D. Weber ${ }^{7}$, Qi Wang ${ }^{2}$, Bärbel Lasitschka ${ }^{10}$, Cynthia C. Bartholomae ${ }^{11}$ Manfred Schmidt ${ }^{11}$, Christof von Kalle ${ }^{11}$, Volker Ast ${ }^{12}$, Chris Lawerenz ${ }^{12}$, Jürgen Eils ${ }^{12}$ Rolf Kabbe ${ }^{2}$, Vladimir Benes ${ }^{13}$, Peter van Sluis ${ }^{14}$, Jan Koster ${ }^{14}$, Richard Volckmann ${ }^{14}$ David Shih ${ }^{15}$, Matthew J. Betts ${ }^{16}$, Robert B. Russell ${ }^{16}$, Simona Coco ${ }^{17}$, Gian Paolo Tonini ${ }^{17}$, Ulrich Schüller ${ }^{18}$, Volkmar Hans ${ }^{19}$, Norbert Graf ${ }^{20}$, Yoo-Jin Kim ${ }^{21}$, Camelia Monoranu ${ }^{22}$, Wolfgang Roggendorf ${ }^{22}$, Andreas Unterberg ${ }^{23}$, Christel Herold-Mende ${ }^{23}$, Till Milde ${ }^{9,24}$, Andreas E. Kulozik ${ }^{9}$, Andreas von Deimling ${ }^{25,26}$, Olaf Witt $^{9,24}$, Eberhard Maass ${ }^{27}$, Jochen Rössler ${ }^{28}$, Martin Ebinger ${ }^{29}$, Martin U.

Schuhmann ${ }^{30}$, Michael C. Frühwald ${ }^{31}$, Martin Hasselblatt ${ }^{32}$, Nada Jabado ${ }^{33}$, Stefan Rutkowski ${ }^{34}$, André O. von Bueren ${ }^{34}$, Dan Williamson ${ }^{35}$, Steven C. Clifford ${ }^{35}$, Martin G. McCabe ${ }^{36,37}$, V. Peter Collins ${ }^{37}$, Stephan Wolf ${ }^{10}$, Stefan Wiemann ${ }^{10,38}$, Hans Lehrach ${ }^{4}$, Benedikt Brors ${ }^{2}$, Wolfram Scheurlen ${ }^{39}$, Jörg Felsberg ${ }^{40}$, Guido Reifenberger ${ }^{40}$, Paul A. Northcott ${ }^{15}$, Michael D. Taylor ${ }^{41}$, Matthew Meyerson ${ }^{6,42}$, Scott L. Pomeroy $6,43^{\prime}$, Marie-Laure Yaspo ${ }^{4}$, Jan O. Korbel ${ }^{3}$, Andrey Korshunov ${ }^{25,26}$, Roland Eils ${ }^{2,44,45 *}$, Stefan M. Pfister ${ }^{1,9} *$ \& Peter Lichter ${ }^{7}$

${ }^{1}$ Division of Pediatric Neurooncology, German Cancer Research Center (DKFZ), Im Neuenheimer Feld 280, Heidelberg 69120, Germany. ${ }^{2}$ Division of Theoretical Bioinformatics, German Cancer Research Center (DKFZ), Im Neuenheimer Feld 280, 
Heidelberg 69120, Germany. ${ }^{3}$ European Molecular Biology Laboratory (EMBL), Meyerhofstrasse 1, Heidelberg 69117, Germany. ${ }^{4}$ Max Planck Institute for Molecular Genetics, Ihnestrasse 63-73, Berlin 14195, Germany. ${ }^{5}$ Division of Child Neurology, Stanford University, 750 Welch Road, Palo Alto, California 94304, USA. ${ }^{6}$ Broad Institute of MIT and Harvard, Cambridge, Massachusetts 02142, USA. ${ }^{7}$ Division of Molecular Genetics, German Cancer Research Center (DKFZ), Im Neuenheimer Feld 280 Heidelberg 69120, Germany. ${ }^{8}$ Department of Neuropathology, NN Burdenko Neurosurgical Institute, 4th Tverskaya-Yamskaya 16, Moscow 125047, Russia. ${ }^{9}$ Department of Pediatric Oncology, Hematology \& Immunology, Heidelberg University Hospital, Im Neuenheimer Feld 430, Heidelberg 69120, Germany. ${ }^{10}$ Genomics and Proteomics Core Facility, German Cancer Research Center (DKFZ), Im Neuenheimer Feld 280, Heidelberg 69120, Germany. ${ }^{11}$ Division of Translational Oncology, German Cancer Research Center (DKFZ) and National Center for Tumor Diseases (NCT), Im Neuenheimer Feld 460, Heidelberg 69120, Germany. ${ }^{12}$ Data Management Facility, German Cancer Research Center (DKFZ), Im Neuenheimer Feld 280, Heidelberg 69120, Germany. ${ }^{13}$ Genomics Core Facility, European Molecular Biology Laboratory (EMBL),

Meyerhofstrasse 1, Heidelberg 69117, Germany. ${ }^{14}$ Department of Oncogenomics, AMC University of Amsterdam, Meibergdreef 9, Amsterdam 1105 AZ, Netherlands. ${ }^{15}$ The Arthur and Sonia Labbatt Brain Tumor Research Centre, Hospital for Sick Children, 555 University Avenue, Toronto, Ontario M5G 1 X8 , Canada. ${ }^{16}$ Cell Networks Cluster of Excellence, University of Heidelberg, Heidelberg 69120, Germany. ${ }^{17}$ Department of Advanced Diagnostic Technologies, IRCCS Azienda Ospedaliera Universitaria San Martino - IST Istituto Nazionale per la Ricerca sul Cancro, L.go R. Benzi,10, Genoa 16132, Italy. ${ }^{18}$ Center for Neuropathology and Prion Research, University of Munich, Feodor-Lynen-Strasse 23, Munich 81377, Germany. ${ }^{19}$ Institute for Neuropathology, Evangelisches Krankenhaus, Remterweg 2, Bielefeld 33617, Germany. ${ }^{20}$ Department of Paediatric Oncology and Haematology, Saarland University Hospital, Homburg 66421, Germany. ${ }^{21}$ Institute for Pathology, Saarland University Hospital, Kirrberger Strasse, Homburg 66424, Germany. ${ }^{22}$ Department of Neuropathology, Institute of Pathology, Würzburg University Josef-Schneider Strasse 2, Würzburg 97080, Germany.

${ }^{23}$ Department of Neurosurgery, Heidelberg University Hospital, Im Neuenheimer Feld 400, Heidelberg 69120, Germany. ${ }^{24}$ Clinical Cooperation Unit Pediatric Oncology, German Cancer Research Center (DKFZ), Im Neuenheimer Feld 280, Heidelberg 69120,
Germany. ${ }^{25}$ Department of Neuropathology, University of Heidelberg, Im Neuenheimer Feld 220, Heidelberg 69120, Germany. ${ }^{26}$ Clinical Cooperation Unit Neuropathology, German Cancer Research Center (DKFZ), Im Neuenheimer Feld 220-221, Heidelberg 69120, Germany. ${ }^{27}$ Department of Pediatric Oncology, Hematology \& Immunology, Klinikum Stuttgart Olgahospital, Bismarckstrasse 8, Stuttgart 70176, Germany. ${ }^{28}$ Department of Paediatric Haematology and Oncology, University Hospital Freiburg, Mathildenstrasse 1, Freiburg 79106, Germany. ${ }^{29}$ Department of Hematology and Oncology, Children's University Hospital, Hoppe-Seyler Strasse 1, Tübingen 72076, Germany. ${ }^{30}$ Department of Neurosurgery, University Hospital, Hoppe-Seyler Strasse 3, Tübingen 72076, Germany. ${ }^{31}$ Children's Hospital Augsburg, Stenglinstrasse 2, Augsburg 86156 , Germany. ${ }^{32}$ Institute of Neuropathology, University Hospital Münster, Albert-Schweitzer-Campus 1, Münster 48149, Germany. ${ }^{33}$ Departments of Pediatrics and Human Genetics, McGill University and the McGill University Health Center Research Institute, Montreal, Quebec H3Z 2Z3, Canada. ${ }^{34}$ Department of Paediatric Haematology and Oncology, University Medical Center Hamburg-Eppendorf, Martinistrasse 52 , Hamburg 20246, Germany. ${ }^{35}$ Northern Institute for Cancer Research, Newcastle University, Royal Victoria Infirmary, Newcastle-upon-Tyne, NE1 4LP, UK. ${ }^{36}$ School of Cancer and Enabling Sciences, University of Manchester, Manchester Academic Health Science Centre, Manchester, M13 9PL, UK. ${ }^{37}$ Division of Molecular Histopathology, Department of Pathology, University of Cambridge, Cambridge CB2 000, UK. ${ }^{38}$ Division of Molecular Genome Analysis, German Cancer Research Center (DKFZ), Im Neuenheimer Feld 280, Heidelberg 69120, Germany. ${ }^{39}$ Cnopf'sche Kinderklinik, Nürnberg Children's Hospital, St-Johannis-Mühlgasse 19, Nürnberg 90419, Germany. ${ }^{40}$ Department of Neuropathology, Heinrich-Heine-University Düsseldorf, Moorenstrasse 5, Düsseldorf 40225, Germany. ${ }^{41}$ Division of Neurosurgery and The Arthur and Sonia Labatt Brain Tumour Research Centre, Hospital for Sick Children, 555 University Avenue, Toronto, Ontario M5G 1X8, Canada. ${ }^{42}$ Dana Farber Cancer Institute, 450 Brookline Avenue, Boston, Massachusetts 02215, USA. ${ }^{43}$ Children's Hospital Boston, 300 Longwood Avenue, Boston, Massachusetts 02115, USA. ${ }^{44}$ Institute of Pharmacy and Molecular Biotechnology, University of Heidelberg, Heidelberg 69120, Germany. ${ }^{45}$ Bioquant Center, University of Heidelberg, Im Neuenheimer Feld 267, Heidelberg 69120 , Germany.

*These authors contributed equally to this work. 